\title{
La gestion du désaccord lors des délibérations en cours d'appel : la politesse stratégique
}

Managing disagreement in a tribunal: the strategic politeness

\section{Rubens Damasceno-Morais}

\section{(2) OpenEdition}

\section{Journals}

Édition électronique

URL : http://journals.openedition.org/tipa/1730

DOI : $10.4000 /$ tipa. 1730

ISSN : 2264-7082

Éditeur

Laboratoire Parole et Langage

Référence électronique

Rubens Damasceno-Morais, « La gestion du désaccord lors des délibérations en cours d'appel : la politesse stratégique», TIPA. Travaux interdisciplinaires sur la parole et le langage [En ligne], 33 | 2017, mis en ligne le 09 octobre 2017, consulté le 26 septembre 2020. URL : http://journals.openedition.org/ tipa/1730 ; DOI : https://doi.org/10.4000/tipa.1730

Ce document a été généré automatiquement le 26 septembre 2020.

\section{(i) $\ominus$

La revue TIPA. Travaux interdisciplinaires sur la parole et le langage est mise à disposition selon les termes de la licence Creative Commons Attribution - Pas d'Utilisation Commerciale - Pas de Modification 4.0 International. 


\section{La gestion du désaccord lors des délibérations en cours d'appel : la politesse stratégique}

Managing disagreement in a tribunal: the strategic politeness

Rubens Damasceno-Morais

\section{Introduction}

1 L'objectif ici est d'analyser des séquences où des magistrats utilisent une construction concessive comme façon de désarmer leur interlocuteur et, grâce à cela, de le pousser à changer d'avis et adhérer à leur opinion ${ }^{1}$. On s'intéressera à la façon dont ces magistrats gèrent le désaccord aux moments du conflit, en arrivant même à "phagocyter» les objections faites par l'interlocuteur réfractaire, dans une cour d'appel brésilienne, à propos d'un jugement fruit d'une affaire médiatique polémique. On ciblera le rôle central que joue alors la concession, en montrant que l'un des magistrats fait « un effort d'être coopératif et poli en exprimant des marques d'accord, en même temps qu'il garde son quant-à-soi » (Doury \& Kerbrat-Orecchioni, 2011).

2 En effet, le mouvement concessif, par nous nommé accord dissonant, a pour fonction d'adoucir le désaccord au moment du délibéré pour que le nœud de la dispute soit défait de façon plus souple: il joue ainsi un rôle stratégiquement irénique. En bref, l'enjeu de l'analyse est de dépasser la description d'occurrences particulières pour exhumer des régularités, et essayer de mettre au jour un phénomène argumentatifinteractif assez curieux. C'est pour cela qu'on va examiner plus d'une séquence, afin de mettre en valeur la régularité du phénomène que l'on a l'intention de dévoiler. En d'autres mots, il s'avère que tous les énoncés de concordance et d'acquiescement du corpus analysé ${ }^{2}$ ne représentent pas effectivement un accord mais que, en fin de compte, cet "accord dissonant " s'avère être un type de désaccord plus inventif car, finalement, dans le fonctionnement pragmatique du discours, le «non» s'habille en 
« oui », montrant que « rien ne se crée ex nihilo dans la parole » (Kerbrat-Orecchioni, $2009: 222)^{3}$.

3 Dans la première partie, sera proposé un parcours théorique à propos des règles et effets de la politesse dans le domaine linguistique de l'analyse des conversations. Dans la deuxième partie (Le langage-en-interaction dans l'univers juridique), on présentera le contexte d'où les données ont été extraites et sera aussi expliqué de façon sommaire le contexte juridique. Finalement, dans la troisième partie (Une affaire médiatique polémique), on proposera une étude de cas pour montrer comment l'accord adouci ou l'«accord dissonant» prend place dans une situation réelle d'interaction entre magistrats dans une cour brésilienne, dans un jugement de dommages et intérêts à propos d'un cas médiatique.

\section{La politesse en contexte polémique}

4 Les études liées à la politesse ont déjà parcouru un chemin important, car on peut leur associer les études de Grice et son principe de coopération (Grice, 1982); les règles du savoir-vivre; les maximes conversationnelles; les conditions de réussite à la Searle (Searle, 1981); les faces négatives suggérées par Brown et Levinson (1987); les territoires et les actes qui les menacent; les actes flatteurs; les rituels et les routines conversationnels; et encore les effets de politesse. À propos des "face work», de la théorie des faces, des règles de politesse, des territoires, et pour une analyse profonde des travaux de Brown et Levinson (1987) et de Goffman (1974), voir notamment les travaux de Kerbrat-Orecchioni (1992, entre autres), Traverso (2007, entre autres), Amossy (2000). On se servira de cette littérature juste pour montrer que, dans les faits, les magistrats dans la délibération analysée sélectionnent dans un vaste répertoire quelques procédés linguistiques qui visent à valoriser leurs collègues de séance, ou du moins à éviter de trop les dévaloriser, dans les moments où le désaccord surgit, et en particulier lors de la situation de désaccord qui se présentera à vif et à chaud.

5 Ces attitudes lors des délibérés confirment la notion même de "politesse " proposée par Kerbrat-Orecchioni (op. cit., 242) et pour l'auteure considérée comme "un phénomène discursif» (ibid.: 241) et qui s'exprimerait à travers des formes grammaticalisées, quelquefois fortement codées, lors des interactions, dans le champ des analyses des conversations. Encore, selon l'auteure: "la politesse est donc un phénomène linguistiquement pertinent " (ibid. : 160). Dans ce contexte la notion de rituel ou de « formule routinisée » est pertinente, bien que, avertit l'auteure, l'étude de ces phénomènes ne doive pas se réduire à quelques formules stéréotypées.

6 Composantes de « la boîte à outils des linguistes », bien que n'étant pas en elles-mêmes de nature linguistique, les règles de politesse, encore selon Kerbrat-Orecchioni, «se font omniprésentes dans tous les types d'échanges, des plus familiers aux plus formels » (ibid. : 241). Et cela nous permet à nous, analystes de l'argumentation dans un contexte d'interaction, de dégager quelques aspects intéressants du fonctionnement des discours (voire des langues), comme on le verra dans les extraits présentés dans la troisième partie de ce travail, où l'idée de politesse se fondra avec ce qu'on considère comme des stratégies argumentatives mises en œuvre par un magistrat pour gagner l'adhésion de ses pairs de délibéré. Au-delà de la « signification sociologique » (Brown \& Levinson, $1987: 1$ ) des actes de politesse, déférence et tact produits dans un contexte institutionnel et fortement ritualisé comme celui qui caractérise les données, on va 
parler ici de la question des attitudes de politesse, essentiellement en raison de leur effet qu'on qualifiera de désarmant ${ }^{4}$. Dans le corpus dont on dispose, ces actes de politesse sans doute acquièrent une valeur rhétorique non négligeable - ce qui rappelle la fable du loup et de l'agneau ${ }^{5}$, comme on le verra. De cette façon, on va essayer de montrer que la politesse, associée à un mouvement réactif concessif des magistrats, et qu'on appellera "l'accord dissonant", permet de masquer d'un voile pudique le moment où un loup en dévore un autre - mais le dévore avec adoucisseurs, gentillesses et beaucoup de finesse.

\section{Le langage-en-interaction dans l'univers juridique}

7 Les phrases et formules qui seront dévoilées dans l'analyse soulignent l'importance du rôle actanciel d'opposant ${ }^{6}$. L'analyse a pour but de montrer que l'entretien dialectique entre les magistrats en délibéré n'est pas une conversation à bâtons rompus ni une discussion " anarchique ». L'échange verbal y est pris dans un réseau de conventions et de règles, qu'il est très éclairant de concevoir sur le modèle des codes institutionnels qui règlementent la pratique d'un sport ou d'un jeu, et qui contraignent, selon des lignes bien définies, le déroulement concret de toute partie réelle ou possible. Ce point nous amène à prendre conscience que ce qui est compris comme relevant de la politesse, en fin de compte, a aussi une fonction institutionnelle, car ceci contribue à nourrir «l'ethos collectif» (Amossy, 2010 : 160), c'est-à-dire l'image attachée au groupe de magistrats et à assurer son bon fonctionnement. En d'autres termes, la manière généralement adoucie de mettre en pratique la gestion du désaccord dans le contexte qu'on va dévoiler est une caractéristique saillante de la façon d'agir des interactants. Elle transparaît à travers des phénomènes multiples : les salutations, les compliments et les excuses, des « rites statutaires ou rites interpersonnels » (Goffman, 1974:51), qui ponctuent les interactions entre les juges, le langage employé, la connaissance parfaite des codes et de la terminologie, la précision des détails et la connaissance des procédures qui nourrissent "l'ethos de compétence juridique et de maîtrise » (Amossy, $2010: 163$, notre soulignement), fondamental, surtout dans un contexte où l'interaction institutionnelle est fortement ritualisée. Sur ce sujet, d'ailleurs, Drew et Heritage (1992 : 43) ont beaucoup à nous apprendre, car ils étudient à fond le contexte juridique sous une optique des rituels d'interaction.

8 À propos de la procédure judiciaire, Dupret (2006 : 229) affirme que «l'activité juridique est avant tout langagière ». Sans ce primat accordé à la dimension langagière de l'activité juridique, on réduirait l'activité judiciaire à de purs raisonnements mathématiques, à des théorèmes ou équations, et toutes les affaires judiciaires seraient réglées par la simple division ou multiplication, sans besoin de recourir au deuxième degré et à ses cours d'appel ou cours de cassation. Un coup de baguette "logique " suffirait et voilà qu'un monde parfait, sans conflits, apparaîtrait. Cependant, le droit appartient au domaine du vraisemblable, du plausible, du probable. Le droit, selon ce que nous apprend Atienza (1997: 17), n'est pas une fin en soi et n'a aucun caractère naturel. Il s'agit plutôt d'une invention humaine que nous devons chercher à façonner et à utiliser intelligemment pour atteindre des objectifs plus généraux, qui vont au-delà de la loi : un peu de paix, une certaine égalité, une certaine liberté...

L'accomplissement du travail juridique se déroule dans un contexte où le juge doit interagir avec un auditoire, composer, dans cette interaction avec les lois, ses propres 
choix, les choix de ses pairs et, surtout, avec le regard de l'opinion publique. L'analyse conversationnelle nous a appris que l'examen d'une décision judiciaire ne peut se faire indépendamment de la façon dont les participants à la procédure s'orientent vers les contraintes propres à l'acquisition et à l'accomplissement de leur savoir (Dupret, 2006 : 164). La mise en œuvre et la négociation de ces contraintes inscrivent toute cette procédure dans le domaine du mouvant, du dynamique, du surprenant et du subjectif, caractéristique de l'univers du langage en interaction. Encore une fois, le droit et le langagier s'entrecroisent.

10 Sans remonter loin dans le temps, il suffit de rappeler, dans cette perspective, les études de Perelman et Tyteca (2008) et leur Nouvelle rhétorique, ainsi que Les usages de l'argumentation de Toulmin (1993), qui ont bouleversé les recherches dans le domaine de l'argumentation langagière liée au domaine juridique, dès la deuxième moitié du XXème siècle. Philosophe du droit, Perelman, par exemple, ne laisse subsister aucun doute sur le fait que c'est la dimension langagière qui constitue l'objet principal des questionnements et d'élaborations philosophiques qui prennent le droit comme objet d'études. "L'argumentation joue un rôle très important dans la loi », souligne Feteris (1999: 1), c'est sans doute ce qui explique que de nombreux théoriciens ancrent leur conception de l'argumentation/rhétorique dans le domaine juridique, d'où ils tirent matière à nourrir leurs réflexions.

11 Dans cet article, la dimension langagière de l'activité juridique sera examinée avec la plus grande attention, dans la mesure où on considère que juger signifie surtout mettre en œuvre la loi avec des mots, des arguments. C'est la pratique langagière qui sera privilégiée par le regard proposé dans cet article ; la perspective adoptée intégrera bien entendu également le domaine de l'argumentation, au sein duquel les études sur le raisonnement juridique sont depuis longtemps en bonne place, à travers notamment l'identification des arguments du type "a pari" et "a contrario", traditionnellement repérés dans le raisonnement juridique, ou à travers des études qui essaient de retracer le parcours des émotions (versus du raisonnement) lorsque les avocats essaient de persuader les juges (tiers au sens plus strict du terme) d'adhérer aux thèses présentées par chaque partie (l'accusation / la défense), dans l'arène des anciens tribunaux jusqu'à nos jours.

12 Nous allons analyser des usages réels de la langue, en situation authentique, en essayant de conjuguer une approche pratique et une réflexion théorique. L'analyse cherche à rendre compte de la façon dont certains mécanismes linguistiques sont mis en œuvre dans un cadre interactionnel, afin de comprendre les liaisons qui s'établissent entre l'énonciatif et l'interactionnel dans une situation argumentative ${ }^{7}$. S'inspirant de cette conception, l'analyse ici présentée soulignera l'importance de la dimension interactionnelle dans le contexte institutionnel envisagé, et montrera quelques conséquences $d u$ fonctionnement du système des tours de parole sur la mécanique du délibéré entre trois magistrats, au moment de leur prise de décision.

Cette approche, qui articule « la grammaire pratique ou praxéologique du faire et du dire en contexte » (Dupret : s.d., 17), est moins préoccupée d'identifier les défaillances de ces pratiques au regard d'un modèle juridique idéal, d'une règle formelle à laquelle elles seraient censées se conformer, que de conduire une réflexion sur les modes de production et l'intelligibilité d'un délibéré dans une Cour d'appel.

14 Nous allons préciser enfin que les énoncés analysés sont intégrés à un type d'interaction trilogal, où trois interactants participent au contexte énonciatif pour 
arriver à la décision judiciaire, à propos du jugement d'une affaire médiatique polémique. Il faut bien le dire, aucune confrontation directe n'a lieu entre les juges pendant le jugement ici analysé - comme il est de mise entre les avocats de l'accusation et ceux de la défense dans une plaidoirie ; la dimension dialogale et la valeur argumentative de la situation présentée seront évaluées à partir d'une série de réactions, (co)orientées avec les paroles du magistrat rapporteur et visant à rendre sensibles les positions soutenues dans le trilogue.

\section{Le cadre institutionnel - une cour d'appel brésilienne}

Dans le domaine du droit de la responsabilité civile, que ce soit dans la législation spécifiquement brésilienne ou dans le système juridique d'autres pays, la question du préjudice moral constitue un sujet épineux ${ }^{8}$. La résolution des conflits qui sont jugés dans ce domaine peut impliquer le versement de dommages et intérêts d'un montant qui doit être déterminé lors d'une procédure civile, en réparation de préjudices moraux ou même physiques. La somme qui sera payée par l'offenseur s'appelle le pretium doloris ou « le prix de la douleur "; il n'est pas rare que son montant fasse l'objet de controverses, soit au moment de la qualification d'un acte comme "préjudice", soit au moment de la définition des critères établissant le montant qui devra être versé comme compensation pour une action considérée comme dommageable, selon les lois civiles au Brésil.

Nous savons que l'univers juridique est strict et formalisé, et que les événements qui s'y déroulent (jugements, délibérations, interrogatoires, etc.) obéissent toujours à des procédures rigides. L'observation attentive des enregistrements du corpus dont on dispose nous a déjà permis de constater que chaque jugement se décompose en deux moments principaux : l'Étape 1, qui est le moment où les magistrats qualifient le fait, et l'Étape 2, où les magistrats parlent du montant qui doit être versé dans le cadre de chaque affaire. Lors de la première étape, les juges décideront, selon les critères offerts par la loi, selon les preuves et les documents présentés à l'occasion du jugement en première instance et enfin selon l'interprétation que les juges feront des affaires si, vraiment, le plaignant a effectivement subi les blessures émotionnelles et psychologiques alléguées. Dans le cas d'une réponse négative, le délibéré arrive à son terme et la question de la valeur du préjudice ne sera pas abordée, sa réalité même n'ayant pas été reconnue. Dans le cas d'une réponse affirmative à la question (lorsque le dommage est reconnu), commence la deuxième étape, au cours de laquelle les autorités auront pour mission de définir le pretium doloris, c'est-à-dire le montant supposé compenser l'offense subie par le plaignant. Maints juristes déclarent que trouver un " prix » pour la douleur représente le moment le plus difficile du délibéré.

Ci-dessous, un schéma illustre les deux étapes du rituel de délibération dans une cour d'appel au Brésil : 


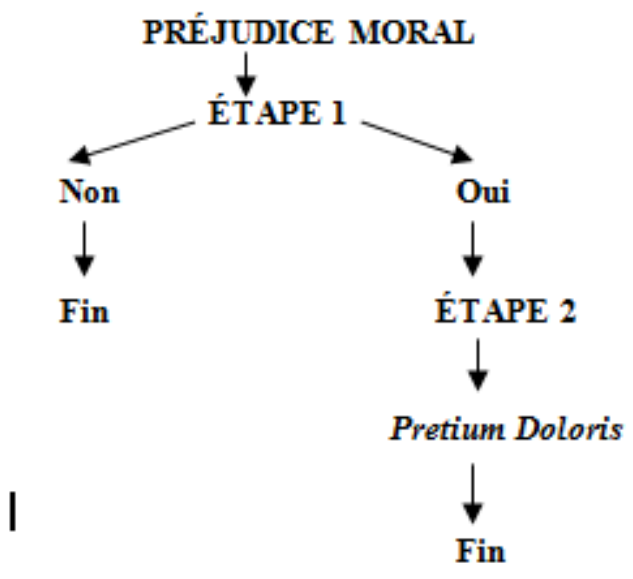

Schéma $1: 2$ étapes dujugement en appel

\section{Une affaire médiatique polémique}

propose ici une étude de cas pour montrer comment l'interprétation d'un magistrat prévaut au détriment du raisonnement de ses collègues, experts eux aussi, à des moments de forte stase argumentative ${ }^{9}$, donc de conflit. On examinera cette affaire, qui a pour objet le jugement d'une entreprise de presse qui avait utilisé des titres 
« injurieux » (selon le demandeur du procès) à la une de son journal ${ }^{10}$. Cette affaire traite, en fin de compte, d'un mécanisme récurrent dans l'expression d'une opinion: l'emploi des adjectifs dans un texte. On le sait, un emploi excessif d'adjectifs dans un texte lui confère une dimension subjective, car il traduit le fait que l'information est présentée de manière orientée (voire tendancieuse). Dans cette affaire, l'existence d'un jugement de valeur est claire dans les titres suivants, publiés dans un journal brésilien de grande renommée : «Les policiers criminels » et "Quand le vilain est la police ». En effet, lors du jugement en première instance, le magistrat considère qu'il y a bien illicéité et condamne le journal à payer, à titre de dommages et intérêts, le montant de 40 mille réaux ${ }^{11}$ aux quatre policiers " outragés » (dix mille pour chaque demandeur du procès). Nonobstant, le procès donne lieu à un nouveau jugement, étant donné que les policiers contestent le montant arbitré par le juge de Première Instance (désormais JPI). Les quatre demandeurs allèguent que le montant de 10 mille réaux pour chacun ne serait suffisant pour «compenser» ni la «douleur morale »qu'ils ont subie, ni les dommages ayant suivi la publication de ces titres. De son côté, l'entreprise responsable du journal qui a publié les titres (le défendeur) fait appel de la décision du JPI, car, selon elle, il n'y a pas d'illicéité dans l'affaire, étant donné que, toujours selon elle, le journal avait le droit de s'exprimer librement.

Dans la première partie du délibéré, c'est-à-dire au moment de la délibération où les magistrats doivent décider s'il existe effectivement une illicéité dans l'affaire (la qualification du fait), les magistrats en délibéré se sont servi des mêmes arguments que ceux qu'avait avancés le JPI pour conclure à l'existence d'un illicite. Le délibéré comporte même des compliments adressés au magistrat de première instance, affirmant que la sentence rendue alors était fort bien justifiée. On présente dans le tableau suivant les principaux arguments utilisés par le juge rapporteur lors de la lecture de son vote; la totalité de la transcription n'est pas reproduite, car il s'agit d'une longue lecture ; cependant, dans les trois extraits qui seront présentés ensuite, on reverra ces arguments qui entrent dans la négociation sur la définition du montant :

\begin{tabular}{|l|l|l|l|l|}
\hline \multicolumn{2}{|l|}{ ARGUMENT (S) ${ }^{12}$} & $\rightarrow$ & CONCLUSION \\
\hline & $\begin{array}{l}\text { les titres des textes publiés dans l'édition du ((date)) montrent à l'évidence que le } \\
\text { journal ((nom)) est allé au-delà du simple } \\
\text { animus narran::di=\& \&=en agissant de façon trop dégradante irresponsable et } \\
\text { offensante pour l'honneur objectif et subjectif des demandeurs } \\
\text { les personnes qui sont accusées ou dénoncées pour un crime supposé ne sont pas } \\
\text { forcément des criminels elles sont tout simplement des suspects et elles ne peuvent } \\
\text { pas juste à cause de cela être stigmatisées comme des vilai:ns des bandi:ts et des } \\
\text { n'importe quoi } \\
\text { devant le grand public } \\
\text { même les gens condamnés gardent le droit à leur } \\
\text { honn:eur surtout l'honneur subjectif et à cause de cela ils ne peuvent pas être est évident } \\
\text { librement diffamés et étiquetés de façon dégradantel }\end{array}$ & l'ilite \\
\hline
\end{tabular}




\begin{tabular}{|l|l|l|} 
C2 & $\begin{array}{l}\text { [le journal] ne s'est pas limité à faire la NARRATION: } \\
\text { des faits mais il QUALIFIE également la conduite des policiers } \\
\text { on est allé au-delà de la simple NARRATION }\end{array}$ & $\begin{array}{l}\text { je n'ai aucun doute } \\
\text { je suis } \\
\text { l'éminent } \\
\text { rapporteur }\end{array}$ \\
\hline $\begin{array}{l}\text { les reportages ne le soient pas il est évident que les titres utilisés pour attirer } \\
\text { l'attention du lecteur ont induit à un jugement négatif envers les policiers }\end{array}$ & $\begin{array}{l}\text { alors je n'ai aucun } \\
\text { doute par } \\
\text { rapport } \\
\text { qualification du } \\
\text { préjudice moral }\end{array}$ \\
\hline
\end{tabular}

Dès que les magistrats, en délibéré, ratifient la décision arbitrée par le JPI, ils se mettent d'accord sur le fait que les titres ont bel et bien porté atteinte au «capital d'honneur » des policiers (Aristote, Rhétorique, livre 1, ch. 9, 1367 b 10). Et, on le sait, une fois le préjudice moral caractérisé et justifié, arrive le moment du jugement sur la définition du montant. Dans cette analyse, on cherche à comprendre pour quelle raison le montant de 15 mille réaux proposé par le deuxième juge conseiller l'a emporté sur les montants proposés par les magistrats, étant donné que chaque expert avait proposé un chiffre différent ( $\mathrm{C} 1$ propose 20 mille, $\mathrm{C} 2,15$ mille et $\mathrm{C} 3,17$ mille et « quelque chose ») et que, comme on peut le voir dans le tableau ci-dessus, les experts ont utilisé pratiquement les mêmes arguments pour justifier la hausse du montant arbitré par le JPI.

De cette façon, est faite l'hypothèse selon laquelle la réponse à cette question ne réside pas vraiment dans le type d'arguments employé par C1, C2 ou C3 ou dans le fait qu'un argument employé par les juges serait plus «fort » ou plus «faible », étant donné que tous les experts (le JPI inclus) ne font que ratifier les arguments les uns des autres. Les trois se sont servi des critères de " raison " et d' « acceptation ${ }^{13}$, donc on ne peut pas affirmer que $\mathrm{X}$ ait utilisé un argument juridiquement plus pertinent que le magistrat $\mathrm{Y}$. La discussion entre les juges dans le deuxième moment du jugement, met une fois encore en évidence que dans le domaine du droit, on ne peut pas parler de raisonnement « correct ou incorrect » (Perelman, 1999:6) et cela témoigne bien de la difficulté que peut représenter, pour les magistrats, le moment de la définition du pretium doloris, malgré les quelques critères proposés par la loi pour traiter cette question.

Après le constat, dans la première partie du délibéré, que les arguments employés par les magistrats se limitent le plus souvent à la ratification des raisons employées par le JPI, on fait l'hypothèse selon laquelle, en réalité, une stratégie interactionnelle (voire rhétorique) est responsable du choix du montant proposé par le premier juge conseiller comme « prix raisonnable » dans cette affaire. Et cela parce qu'on croit réellement qu'il est important d'articuler l'analyse du langage juridique avec les études rhétoriques. Selon Meyer (1999 : 5, notre soulignement) « que ce soit le droit ou la politique, la philosophie ou l'analyse littéraire, rien n'échappe à la rhétorique ", même si les juristes n'aiment pas vraiment cette idée. Et leur crainte s'explique peut-être par l'idée que toute rhétorique vise à tendre un piège.

En effet, comme on l'avait remarqué précédemment, l'analyse des négociations entre les magistrats lors de la deuxième partie des délibérés montre d'intéressants 
phénomènes argumentatifs-interactionnels qui n'apparaissent pas dans le document produit après le délibéré. Cela montre bien qu'on ne peut jamais réduire l'analyse argumentative à la simple analyse d'un raisonnement mathématique ou de propositions logiques qui ignoreraient le côté humain, dialogique et interactionnel du langage en action; on considère ici que «l'argumentation est [aussi] un produit de l'interaction » (Forget, $1994:$ 71).

Faute de place pour analyser en détails le parcours interactionnel des trois magistrats, on va cibler les réactions et le comportement du premier juge conseiller/C1 (qui joue en même temps le rôle institutionnel de réviseur du procès) lors de la négociation entre les experts pour la définition du montant de cette affaire ; c'est en effet le "prix " suggéré par C1 qui l'a emporté sur les chiffres proposés par les autres magistrats.

Voyons la première séquence proposée :

\section{SÉQUENCE 1}

\begin{tabular}{|c|c|c|}
\hline & & (L'affaire du titre offensant) \\
\hline 83 & C1 & $<(($ changement du ton de la voix $))$ il arrive \\
\hline 84 & & que $>$ le ((identification)) comme il avait été bien remarqué dans \\
\hline \multirow[t]{2}{*}{85} & & la sentence et à présent lors du vote du éminent rapporteur les \\
\hline & & titres ne se sont pas limités à faire \\
\hline \multirow[t]{2}{*}{86} & & la QUALIFICATION: de du fait mais ils font aussi toute une \\
\hline & & ADJECTIVATION de la conduite des agents moi j'e n'ai rien \\
\hline 87 & & à reprocher à la justification de la sentence et au vote de \\
\hline 88 & & l'éminent rapporteur et pour cette raison euh je suis d'accord \\
\hline 89 & & avec lui cependant par rapport au \\
\hline 90 & & au par rapport \\
\hline \multirow[t]{2}{*}{91} & & par rapport cependant à cette valeur je \\
\hline & & voudrais avant de conclure mon vote de: (2s) pondérer avec \\
\hline 92 & & l'éminent rapporteur pour qu'on reste \\
\hline 93 & & dans le moyen terme n'est ce pas/seulement la qualification \\
\hline 94 & & mise en évidence et-et: la façon qu'elle avait été rédigée et \\
\hline \multirow[t]{2}{*}{95} & & pour cela euh le titre est sans doute offensant \\
\hline & & et encore plus offensant à cause des adjectifs employés euh cela \\
\hline 96 & & procure sans aucun doute un \\
\hline
\end{tabular}




\begin{tabular}{|c|c|}
\hline \multirow[t]{2}{*}{97} & préjudice moral mais comme la jurisprudence à maintes reprises \\
\hline & demande aux juges qu'ils soient modérés: dans l'évaluation du \\
\hline 98 & dédommagement n'est ce pas/ et-et: il y a eu \\
\hline 99 & effectivement une poursuite pénale et: ils ont été \\
\hline 100 & acquittés même par manque de preuves \#etc\# votre honneur \\
\hline 101 & voyez vous la possibilité de baisser ce montant/d'arriver \\
\hline & 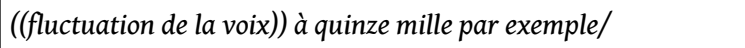 \\
\hline
\end{tabular}

Dans cette séquence, on voit que le premier juge conseiller/C1, au moment d'annoncer son vote, manifeste son adhésion au vote qui vient d'être prononcé par le juge rapporteur (1.83 à 88). Nonobstant, cet assentiment, en réalité, annonce un désaccord, ce qui rappelle les structures concessives du type : « en disant $p$, tu serais en droit de tirer la conclusion $\mathrm{r}$; mais il ne faut pas, car il existe un argument $\mathrm{q}$, plus fort que $\mathrm{p}$, qui mène à non-r » (Moeschler \& Spengler, $1982: 10)$. Quand C1 affirme que "moi je n'ai rien à reprocher à la justification de la sentence et au vote de l'éminent rapporteur " (1.86-88), ce magistrat oriente son raisonnement vers une conclusion du type «Je suis d'accord avec Votre Honneur, moi aussi je propose le montant de 20.000 » (le montant qui vient d'être proposé par le rapporteur). Cependant, il existe un argument $q$ ("seulement la qualification mise en évidence », 1.93-94) qui « anti-oriente » la conclusion qui se dessinait, conduisant finalement à une conclusion non-r (la fixation du montant à 15000 réaux, en opposition aux 20000 proposés par le rapporteur).

En effet, la notion d' " anti-orientation » est depuis longtemps présente dans les études de sémantique chez Ducrot (1995 : 145-165). L'idée de « coup argumentatif anti-orienté » est bien travaillée aussi dans l'article de Doury et Kerbrat-Orecchioni (2011), où les auteures rappellent à propos de la structure de la concession : «q est généralement introduit par un connecteur oppositif (classiquement, mais)». Ce deuxième argument est présenté comme l'emportant sur le premier (le mouvement concessif permet donc de conclure à non-r). Dans leur étude, les auteures ajoutent que la concession serait une unité argumentative complexe étudiée dans le domaine de la contre-argumentation. Moeschler et Spengler, dans leur étude plus ancienne sur la concession, parlent déjà de « conclusion inverse non-r » (1982:13).

31 Il est encore à noter que $\mathrm{C} 1$, en adoptant ce positionnement concessif au moment de la négociation avec le juge rapporteur, continue à manifester son assentiment vis-à-vis du vote de son interlocuteur, quand il dit : " et pour cela euh le titre est sans doute offensant et encore plus offensant à cause des adjectifs employés euh cela procure sans aucun doute un préjudice moral » (1.94-97), et, une fois encore, ce magistrat présente ainsi un argument $q$ comme plus fort que $p$ (« mais comme la jurisprudence à maintes reprises demande aux juges qu'ils soient modérés: dans l'évaluation du dédommagement n'est-ce pas », 1.97-98), argument qui conduit à une conclusion non-r (les 15 mille réaux proposés par ce juge à la ligne 101). La structure concessive de ce jeu argumentatif est représentée dans le tableau cidessous : 
Tableau sur la concession argumentative dans les extraits présentés

\begin{tabular}{|c|c|c|c|}
\hline \multicolumn{2}{|l|}{ Orientation } & \multicolumn{2}{|l|}{ Anti-orientation } \\
\hline $\mathrm{p}$ & conclusion $r$ & q & $\begin{array}{l}\text { conclusion } \\
\text { non-r }\end{array}$ \\
\hline $\begin{array}{l}\text { moi je n'ai rien à reprocher à } \\
\text { la justification de la sentence } \\
\text { et au vote de l'éminent } \\
\text { rapporteur et pour cette raison } \\
\text { euh je suis d'accord avec lui (l. } \\
86-89 \text { ) }\end{array}$ & $(20000)^{14}$ & $\begin{array}{l}\text { seulement la qualification mise en } \\
\text { évidence et-et: la façon qu'elle } \\
\text { avait été rédigée (93-94) }\end{array}$ & 15000 \\
\hline $\begin{array}{l}\text { et pour cela euh le titre est } \\
\text { sans doute offensant et encore } \\
\text { plus offensant à cause des } \\
\text { adjectifs employés (1.94-95) }\end{array}$ & \multirow{2}{*}{$\begin{array}{l}\text { euh cela procure } \\
\text { sans aucun doute } \\
\text { un préjudice } \\
\text { moral (1.95-97) }\end{array}$} & \multirow{2}{*}{$\begin{array}{l}\text { mais comme la jurisprudence à } \\
\text { maintes reprises demande aux } \\
\text { juges qu'ils soient modérés: dans } \\
\text { l'évaluation du dédommagement } \\
\text { n'est-ce pas (1.9 7-98) }\end{array}$} & \multirow[b]{2}{*}{15000} \\
\hline $\begin{array}{l}\text { et-et: il y a eu effectivement } \\
\text { une poursuite pénale et: ils ont } \\
\text { été acquittés même par } \\
\text { manque de preuves \#etc\# } \\
(1.98-100)\end{array}$ & & & \\
\hline
\end{tabular}

Lors des deux prochaines séquences de cette même affaire (Séquences 2 et 3), on va montrer que cette stratégie « concessive » est maintenue par le premier juge conseiller pour la négociation avec le juge rapporteur au moment de la définition du montant. Cependant, il semble que dans les exemples qui vont suivre, la structure concessive à proprement parler n'apparaisse plus, du moins pas sous la forme qu'on vient de mettre en évidence dans la Séquence 1 reproduite ci-dessus. Le mouvement concessif sera appelé « accord dissonant », comme on va le voir à présent.

\section{SÉQUENCE 2}

\begin{tabular}{|l|l|l|}
\hline & & (L'affaire du titre offensant) \\
\hline 56 & C1 & je donne la parole à l'éminent rapporteur \\
\hline 57 & RAP & $(($ reprend la parole, hésitant)) en réalité je crois que euh: le \\
\hline 58 & & ((identification)) a mal agi par rapport aux titres du texte \\
\hline 59 & & n'est ce pas/ quand \\
\hline 60 & C1 & [ils les qualifient (.) de (.) malfaiteurs\& \\
\hline 61 & RAP & \&comme des policiers corrom:[pus (.) il porte un jugement\& \\
\hline
\end{tabular}




\begin{tabular}{|l|l|l|}
\hline 62 & C1 & [oui: 'exactement $^{\circ}$ ' \\
\hline 63 & RAP & \&de valeur dépréciatif aux-aux gens aux demandeurs \\
\hline 64 & & \#défendeurs\# \\
\hline 65 & C1 & moi-moi je ferais juste une pondération par rapport au-au \\
\hline 66 & & montant de ce préjudice làl $=$ \\
\hline 67 & RAP & $=\# c^{\prime}$ est une suggestion\# président (.) on es[t \& \\
\hline 68 & C1 & ['n'est ce pas ${ }^{\circ}$ \\
\hline 69 & RAP & \&ouvert au dialogue \\
\hline 70 & & $(2$ sec) \\
\hline
\end{tabular}

Dans cette nouvelle séquence de l'affaire, on voit que le premier juge conseiller/C1, surtout dans les lignes 60 et 62, manifeste un appui total au raisonnement du juge rapporteur (“je n'ai aucun doute par rapport» / " oui: 'exactement ${ }^{\circ}$ \) ainsi qu'à la conclusion $r$ présentée par ce dernier à propos de l'existence d'un illicite dans l'affaire en jugement. À ce moment, on pourrait dire que le rapporteur et le réviseur partagent une même schématisation ou « représentation discursive » (Grize, 1996 : 50). Nonobstant, dans les lignes 65-66, dans un coup anti-orienté, le réviseur/C1 suggère un autre prix, plus bas, ce qui, d'une certaine façon, atteste déjà de la présence d'un conflit (une conclusion non-r) entre $\mathrm{C} 1$ et le vote du rapporteur, malgré les manifestations d'assentiment produites par $\mathrm{C} 1$. Cette ouverture au débat montrée par $\mathrm{C} 1$ semble bien relever de la politesse, et viser à "désarmer " l'interlocuteur, pour reprendre les termes employés par Kerbrat-Orecchioni (1992:301). Ce serait en fin de compte une façon pour $\mathrm{C} 1$ d'exhiber un ethos positif, sympathique et surtout bienveillant au moment précis du désaccord ferme, pour que le désaccord imminent (la proposition d'un autre montant) soit reçu sans objection par le juge rapporteur (et c'est précisément ce qui va se passer dans la séquence suivante). Bref, comme on va s'efforcer de le montrer tout de suite, le premier juge conseiller insiste sur cette stratégie qui consiste à énoncer des mots "d'appui » à son interlocuteur, précisément au moment où il n'est pas du tout d'accord avec lui, lors de la définition du pretium doloris. Ce procédé est plus net encore dans le prochain extrait, où les magistrats continuent le duel pour la définition de la valeur du dédommagement. Voyons comment cela se passe :

\section{SÉQUENCE 3}

\begin{tabular}{|l|l|l|}
\hline & & (L'affaire du titre offensant) \\
\hline 102 & RAP & $=$ ah je crois que le montant proposé par votre honneur comme \\
\hline 103 & & celui que je propose respecte le principe de la \\
\hline
\end{tabular}




\begin{tabular}{|l|l|l|}
\hline 104 & & proporcionali[da:de\& \\
\hline 105 & C1 & [exactement \\
\hline 106 & RAP & \&de la razoabilida:del $=$ \\
\hline 107 & C1 & $=$ de la razoabilida:del \\
\hline 108 & RAP & eeuuh: ((hésitant) $)$ \\
\hline 109 & C1 & ((voix catégorique)) quinze mille/ votre honneur xxx= \\
\hline 110 & RAP & $=$ okj'augmente à quinze mille réaux \\
\hline 111 & C1 & '\#ça marche\# (.) alors par rapport au montant du dédommagement \\
\hline 112 & & moi je n'ai rien à ajouter j'accompagne l'éminent rapporteur \\
\hline 113 & & $<(($ changement du ton de la voix)) comment vote le juge ((à C2))> \\
\hline
\end{tabular}

Il est intéressant de remarquer que la conclusion non-r (15 000) proposée par C1 est d'abord refusée par le juge rapporteur, quand celui-ci dit : «=ah je crois que le montant proposé par votre honneur comme celui que je propose » (1.102-103), ce qui caractérise une ambiance un peu conflictuelle, étant donné qu'il y a un désaccord explicite par rapport aux montants du préjudice moral en débat. Et à ce moment de l'interaction, C1, d'une façon réactive, devant le refus du rapporteur, manifeste à nouveau, de façon très adoucie, son "adhésion » à la parole du juge rapporteur, alors qu'en réalité, C1 est en train de suggérer un montant plus bas $(15000)$ que celui que vient de proposer le rapporteur $(20000)$. Ainsi on voit que le juge réviseur (C1), une fois encore, insiste sur sa conclusion non-r (15 000). On qualifie de rhétorique la façon de réagir de ce juge parce que C1, lorsqu'il manifeste de façon marquée son " accord ", réagit dans le but de nous faire croire qu'il voulait tout simplement défaire la stase qui s'était présentée entre lui et son interlocuteur à propos du montant quand il énonce avec chaleur « [exactement» (1.105).

La même chose se passe aussi quand il fait écho, cette fois encore avec chaleur, à un mot qui vient d'être prononcé par le juge rapporteur («=de la razoabilida:de\», 1.107). À son tour, le juge rapporteur, apparemment surpris par l'attitude réactive du juge réviseur (voir 1.109), énonce : « =ok j'augmente à quinze mille réaux» $(1.110)^{15}$, alors qu'en réalité, il est en train de baisser le montant, car, on l'a vu, la somme par lui proposée était de 20000 réaux, et non de 15000 ! Ainsi, dès que le juge rapporteur, bouleversé par l'offensive dynamique de prise de tour menée par C1, accepte de revoir à la baisse le montant qu'il a proposé quelques secondes plus tôt, il finit par capituler devant son interlocuteur, poussé qu'il est de donner une conclusion non-r à son raisonnement (15 000 réaux au lieu des 20000 qu'il avait proposés).

Il nous semble que c'est grâce à cette façon ingénieuse, affable et furtive qu'a C1 d'intervenir dans la négociation avec le juge rapporteur (dont on sait qu'il n'est pas du tout d'accord avec le montant proposé par son interlocuteur) que $\mathrm{C} 1$ amène RAP à changer d'avis. C'est cette stratégie adoptée par C1 qu'on appellera la stratégie de l'accord dissonant. Elle présente quelques caractéristiques de la concession (voir la 
Séquence 1, analysée précédemment), puisqu'elle satisfait à une « double exigence de l'interaction : être coopératif et poli » (Doury \& Kerbrat-Orecchioni, $2011: 5$ ); mais elle s'en éloigne pour une raison principale : la structure présentée n'adopte pas la forme classique de la concession, dans les deux dernières séquences présentées. Et là, il faut rappeler que, selon Vincent et Heisler, " la structure concessive contient quatre constituants : l'argument de départ que nous appellerons la thèse $t$, l'élément concédé c, l'énoncé de rejet de la concession -c et la réaffirmation de la thèse $r$ (que certains appellent la conclusion) $)(1999: 24)$.

Si l'on s'intéresse à l'interaction qui se déploie dans un contexte argumentatif, l'analyse des deux dernières séquences sont intéressantes en ce qui concerne les invasions de tour par le premier juge conseiller, car elles corroborent une fois encore la tournure surprenante et en même temps très dynamique de la stratégie utilisée par ce magistrat. En agissant ainsi, en effet, le premier juge conseiller déclenche la conclusion non-r finalement adoptée par le juge rapporteur «malgré lui » (1.111), puisque comme on l'a vu, c'est le montant de 15000 réaux qui sera adopté par le rapporteur au lieu des 20000 qu'il avait défendus tout au long du délibéré. Ce qu'on essaie de montrer par cette petite étude de cas (Séquences 1, 2, 3), c'est que les invasions de tour menées par C1, toujours très affables mais fermes, montrent la force et la sûreté de la stratégie du juge réviseur qui, grâce à cette dynamique d'« invasion adoucie » de l'espace discursif de son interlocuteur rapporteur, ne lui laisse pas un temps de réflexion assez long pour chercher à imposer le montant qu'il comptait défendre au départ ( 15 mille réaux, au lieu de 20 mille). Le juge rapporteur a ainsi été déconcerté par l'intervention souple mais en ferme opposition, du juge réviseur; c'est peut-être pour cette raison que le rapporteur n'a pas eu le temps de mettre en place la défense du montant par lui proposé et attaqué par le premier juge conseiller. Ainsi, au lieu de conclure son raisonnement en proposant 20000 réaux (c'est-à-dire en soutenant la conclusion $r$ par le biais des arguments par lui employés), le rapporteur se rallie aux 15000 réaux (conclusion non-r soutenue par le premier juge conseiller). Bref, l'accord dissonant s'affiche encore une fois, dans l'élocution de C1 $(1.105,107)$, par l'anticipation d'un veto (ou d'une conclusion non-r) mais toujours sous la forme d'un assentiment emphatisé, d'un accord factice : un loup en habit d'agneau! C'est pourquoi, même en présentant les mêmes arguments techniques que ses collègues experts (le juge rapporteur et le deuxième juge conseiller), c'est grâce à cette façon, docile et ferme, d'agir lors de l'interaction que le premier juge conseiller/C1 arrive à (con)vaincre ses collègues de délibéré et à faire prévaloir au final le montant de 15 mille réaux (et non les autres montants proposés) au titre du " prix » qui sera officialisé à titre de dédommagement à la fin de ce jugement.

Conclusion

Lors de l'analyse présentée, le contenu des extraits nous a montré le moment où un magistrat s'adresse à son interlocuteur de façon stratégique, donc "potentiellement persuasive " (Aristote, Rhétorique, Livre 1, chapitre 2, 1355 b 26). Nous avons vu que cette attitude lors du délibéré confère une dimension rhétorique aux négociations à propos du pretium doloris. En effet, bien que ces interactions n'aient pas de visée argumentative avouée (dans la mesure où chaque magistrat n'est pas censé convaincre l'autre d'adhérer à son vote), nous avons observé que les locuteurs construisent des stratégies pour faire prévaloir leur conclusion (le montant à verser) au détriment de celles de leurs collègues de séance, en particulier dans les affaires les plus polémiques. Ainsi, la richesse des interactions du corpus dont nous disposons permettra de dégager 
une galerie de masques ou une garde-robe de costumes correspondant à une multitude de rôles et de stratégies différents joués par les interactants ${ }^{16}$, c'est-à-dire les juges experts.

A été, ainsi, examiné de plus près les procédés d'adoucissement du désaccord exprimé par un magistrat vis-à-vis de l'avis émis par ses pairs en délibéré. Nous avons essayé cette fois de mettre en relief la dimension stratégique de l'adoucissement lors du jugement d'une affaire médiatique. Notre but était d'explorer la façon dont les magistrats prennent en charge la gestion du désaccord dans les moments de conflit, et en viennent même à " phagocyter » les objections faites par l'interlocuteur réfractaire. En effet, le mouvement concessif qu'on a appelé accord dissonant a pour mission d'adoucir le désaccord au moment du délibéré pour que le nœud de la dispute soit défait de façon plus souple : stratégiquement irénique.

\section{BIBLIOGRAPHIE}

Amossy, R. (2000) L'argumentation dans le discours - discours politique, littérature d'idées, fiction, Paris: Nathan Université.

Amossy, R. (2010) La présentation de soi - Ethos et identité verbale, Paris: Presses Universitaires de France.

Aristote (2007) Rhétorique, présentation et traduction par Pierre Chiron, Paris : GF Flammarion.

Atienza, M. (1997) Derecho y argumentacion, Universidad Externado de Colombia (Serie de Teoria Juridica y Filosofia del Derecho, 6).

Brown, P. \& S. C. Levinson (1987) Politeness - Some universals in language use, Cambridge, UK: University Press.

Doury, M. \& C. Kerbrat-Orecchioni (2011) La place de l'accord dans l'argumentation polémique : le cas du débat Sarkozy/Royal (2007), A contrario [en ligne], 16, 2. Disponible sur : http:// www.cairn.info/revue-a-contrario-2011-2.htm (consulté le 26 juin 2017).

Drew P. \& J. Heritage (eds.), (1992) Talk at work: interaction in institutional settings, Cambridge, UK: Cambridge University Press.

Ducrot, O. (1989) Logique, structure, énonciation, Paris : Minuit.

Ducrot, O. (1995) Les modificateurs déréalisants, Journal of Pragmatics, 24, p. 145-165.

Dupret, B. (2001) L'intention en acte. Approche pragmatique de la qualification pénale dans un contexte égyptien, Droit et société, 2, 48, p. 439-465.

Dupret, B. (2006) Le jugement en action - ethnométhodologie du droit, de la morale et de la justice en Égypte, Genève/Paris: Librairie Droz.

Feteris, E. T. (1999) Fundamentals of legal argumentation - a survey theories on the justification judicial decisions, Dordrecht, Netherlands: Kluwer Academic Publishers. 
Forget, D. (1994) Anticipation et argumentation: la prolepse, Revue québecoise de linguistique, 23, 1, p. 61-67.

Goffman, E. (1974) Les rites d'interaction, Paris: Minuit.

Grice, H. P. (1982) Lógica e conversação, traduit de l'anglais par João Wanderley Geraldi, in Dascal, M. (Org.), Fundamentos metodológicos da linguística, 4, Pragmática. Campinas, Brasil (Coleção Fundamentos Metodológicos da Linguística).

Grize, J.-B. (1996) Logique naturelle et communication, Paris: Presses Universitaires de France. Kerbrat-Orecchioni, C. (1992) Les Interactions Verbales, 2, Paris: Armand Colin.

Kerbrat-Orecchioni, C. (2009) L'énonciation - de la subjectivité dans le langage, 4ème éd., Paris: Armand Colin.

Meyer, M. (ed.) (1999) Histoire de la Rhétorique des Grecs à nos jours, Paris: Librairie Générale Française.

Moeschler, J. \& N. Spengler (1982) La concession ou la réfutation interdite - approches argumentative et conversationnelle, Cahiers de linguistique française, 4, p. 7-36.

Perelman, C. (1999) Logique juridique - Nouvelle rhétorique, Paris: Éditions Dalloz.

Perelman, C. \& L. Olbrechts-Tyteca (2008) Traité d'argumentation, Bruxelles: Éditions de l'Université de Bruxelles.

Plantin, C. (1990) Essais sur l'argumentation, Paris: Kimé.

Plantin, C. (2016) Dictionnaire de l'argumentation, Lyon : ENS Editions.

Reis, C. (2010) Dano moral, $5^{a}$ edição ampliada, Rio de Janeiro : Editora Forense. Searle, J. (1981) Os atos da fala, Coimbra: Almedine.

Toulmin, S. E. (1993) Les usages de l'argumentation, traduit de l'anglais par Philippe de Brabanter, Paris: Presses Universitaires de France (L'Interrogations Philosophique).

Traverso, V. (2007) L'analyse des conversations, Paris: Armand Colin.

Vincent, D. \& T. Heisler (1999) L'anticipation d'objections : prolepse, concession et réfutation dans la langue spontanée, Revue québécoise de linguistique, 27, 1, p. 15-31.

\section{NOTES}

1. Cette analyse fait partie d'un extrait de la thèse de doctorat « Le prix de la douleur : Gestion des désaccords entre magistrats, dans un tribunal brésilien de seconde instance ", soutenue à Lyon 2, en juillet/2013. Une version initiale de cette analyse est aussi parue en portugais, dans le livre « $A$ construção da opinião na mídia », publié au Brésil en 2013, sous forme d'un chapitre.

2. On analyse le corpus TRIBUNAL, enregistré dans une cour d'appel brésilienne à partir duquel on a transcrit les paroles des magistrats à vif et à chaud, lors de quelques délibérés assez polémiques.

3. Dans un autre ouvrage, l'auteure, à propos des mouvements concessifs dans le discours, parle de « camouflage poli du désaccord » (Kerbrat-Orecchioni, 1992 : 245).

4. Nous sommes tout à fait d'accord avec Kerbrat-Orecchioni, quand elle affirme que «la politesse est désarmante » (1992: 301, notre soulignement). 
5. Dans le Tome II de ses Interactions Verbales, Kerbrat-Orecchioni écrit : « la politesse est ce qui fait que l'homme n'est pas un loup pour l'homme» (p. 318). Molière, et son Misanthrope, ne disait pas le contraire!

6. Selon Plantin (1990), les « rôles actantiels » qu'une personne pourrait exercer dans une argumentation sont le Proposant, l'Opposant et le Tiers. En somme, ce sont les positions possibles qu'incarnent les participants dans un débat. Dans un contexte juridique, par exemple, rien n'empêche qu'un même juge, dans le même tour de parole, incarne les positions de proposant et d'opposant.

7. Selon Traverso : « $[\mathrm{u}] \mathrm{n}$ des principes de l'interactionisme est que le langage doit être étudié en situation » $(2007: 17)$.

8. Comme il ne s'agit pas d'une étude du droit comparé (le droit brésilien versus le droit français), les ouvrages ici référencés expliquent le " préjudice / dommage moral » selon la législation brésilienne, bien qu'il ne soit pas rare qu'ils fassent référence au code civil français. Pour en savoir plus : Clayton REIS, Dano Moral (2010) ; Yussef SAID CAHALI, Dano Moral (2005) ; Eduardo de Oliveira LEITE, Grandes Temas da Atualidade : Dano Moral (2002) ; M. Francisca CARNEIRO, Avaliação do Dano Moral (1998), pour ne signaler que quelques ouvrages récents sur le sujet.

9. Selon Plantin (à paraître), « la notion de 'question argumentative' a son origine dans la notion de stase, développée pour l'interaction judiciaire, théorisée par l'argumentation rhétorique ». L'idée de stase naît d'une notion technique issue de l'univers de la médecine, qui traduit le blocage de la bonne circulation des fluides humains et qui, transposée au monde de l'argumentation et de l'interaction, renvoie au conflit, au désaccord, aux différends et divergences entre parties lors d'une discussion

10. Sur l'analyse de titres comme discours ou comme unité de l'organisation textuelle, voir la thèse de Wander Emediato de Souza, Analyse des configurations linguistiques et discursives des titres de journaux français et brésiliens, soutenue à l'Université de Paris XIII en février 2000.

11. Réal - la monnaie brésilienne.

12. Il faut garder en mémoire qu'il s'agit d'un texte oral, spontané ; de cette façon, il y a des mots qui manquent, des mots incomplets, des phrases non terminées etc. On fait ici juste une version du texte en portugais, lequel, lui même, n'est pas toujours totalement clair. Voilà les conventions de transcription adoptées : / intonation montante ; \intonation descendante ; (.) pause brève ; (..) pause moyenne ; (..) pause longue ; $(0.6)$ pause en secondes ; [ ] chevauchement : xxx segment inaudible ; ((rit)) commentaire ; ( ) transcription incertaine ; \& absence d'intervalle entre deux tours ; = continuation du même tour ; VIdéo emphase ; : allongement ; - interruption ; ${ }^{\circ}$ voix basse ; ${ }^{<}(())$bla bla` ; \# voix accélérée.

13. En langage juridique, les magistrats parlent du critère de la « razoabilidade » (le fait d'être un argument raisonnable) et du critère de l'« acceptation ", c'est-à-dire, le critère de l'acceptation d'un argument.

14. Cette conclusion est juste une hypothèse dans notre analyse, car $\mathrm{C} 1$ propose en effet 15 mille réaux.

15. Il est intéressant de remarquer que l'enregistrement sonore nous fait entendre une prononciation embarrassée, lorsque le rapporteur accepte le montant revendiqué par C1.

16. On paraphrase ici délibérément Ducrot, quand il dit: « le trésor de phrases mis à notre disposition par la langue est en même temps une galerie de masques ou une garde-robe de costumes permettant de jouer une multitude de personnages différents » (1989: 173). 


\section{RÉSUMÉS}

Dans cet article, on poursuivra l'observation du caractère adouci du désaccord exprimé par quelques magistrats vis-à-vis de leurs pairs dans un délibéré, dans une cour d'appel brésilienne, où on juge une affaire concernant un fait médiatique politique (L'Affaire des titres offensants). On essayera de mettre en relief le côté stratégique des paroles polies et adoucies employées par les magistrats lors du délibéré. On verra que de telles attitudes lors des délibérés manifestent la dimension rhétorique des négociations à propos du pretium doloris. En effet, bien que ces interactions n'aient pas de visée argumentative avouée (chaque magistrat n'étant pas censé convaincre l'autre d'adhérer à son vote), on a observé qu'à certains moments, les participants construisent des stratégies pour faire prévaloir leur conclusion (le montant à verser) au détriment de celles de leurs collègues de séance. De cette façon, on prétend inspecter la façon dont les interactants prennent en charge la gestion du désaccord aux moments de conflit, arrivant même à "phagocyter » les objections faites par l'interlocuteur réfractaire. En effet, le mouvement concessif, par nous nommé accord dissonant, et qui sera montré en détails dans l'analyse présentée a pour mission d'adoucir le désaccord au moment du délibéré pour que le nœud de la dispute soit défait de façon plus souple: stratégiquement irénique. En somme, l'intérêt stratégique que peut avoir le mouvement concessif dans les données dont on dispose n'est pas anodin, comme on le montrera.

This article studies the nature of a disagreement expressed by some judges to their peers in a deliberative judgment, in a Brazilian court of appeal, where three magistrates analyse a legal affair involving a political media fact. The proposed analysis was to inspect linguistic structures where magistrates use a concessive construction as a way to disarm their interlocutor and to push his colleagues to change their mind and join their standpoint. The major interest here was to observe how those judges manage disagreement (stasis) when the polemic is installed during the complex deliberation by magistrates. Our data showed some interesting moments when one magistrate "drag" the objections made by his refractory interlocutor, in a Brazilian court of appeals. The analysis shows the central role of the concessive linguistic structure in that debate and the rhetoric effects caused by that use, confirming that, in a debate, sometimes, people "make an effort to be cooperative and polite in expressing agreement marks, at the same time that they keeps his dignity" in a strategic way (Doury and Kerbrat-Orecchioni, 2011). Indeed, the concessive linguistic and rhetorical movement, that we called, in this work, the "dissonant agreement", occurs in that context mainly to mitigate the disagreement in the deliberation table. This way, as we observed, the most important moment of the argumentation is strategically defeated in a more flexible/smooth way, without became a classical polemical issue, during the deliberation, because of the called dissonant agreement. The main issue of the analysis was to go beyond a simple description of a specific occurrence (the use of concessive structures) to demonstrate regularities in that kind of argumentative interaction and try to update a curious rhetorical and inventive phenomenon. That's why we proposed here to examine more than one concessive linguistic structure, in order to highlight the regularity of the phenomenon that the analysis intends to disclose (i.e. dissonant agreement). In other words, we realized that some statements of agreement and acquiescence of the data analysed did not represent an effective consensus or an acceptance of the other's standpoint. Effectively, we observed that this way of disagreement, that is, in saying "yes" when someone would like to say "non", looks more inventive and strategic, because, in that way, someone apparently avoid a possible frontal combat between different standpoints. This strategy demonstrates that "nothing is created ex nihilo in the language" (Kerbrat-Orecchioni, $2009: 222$ ). And this ascertainment is really 
appropriate in this work about a deliberation in a tribunal, because in the examined legal deliberation ("The insulting title's case") we could observe the way that sentences in a deliberation mean more than the words apparently try to say; we were able to observe some ways to win a debate in a subtle way, in a legal territory among magistrates. In addition, the trial shows a recent political situation in the recent Brazilian history, what, we guess, might increase the interest for the analysis, because we examine a cultural side, in a situated context. On the other side, it must be said that this case does not represent the general Brazilian political life, but it gives an idea about the headlines that have been visiting newspapers during the last years. In the first part of the article, we propose a theoretical explanation about the rules and effects of politeness in the linguistic field of the Analysis of Conversation. With the help of authors like Grice (1982), Searle (1981), Kerbrat-Orecchioni (1992), among others, we will find theoretical support to help us to structure the proposed analysis. In the second part (the language-ininteraction in the legal universe) we present the context from which the data was extracted and, also, we explain summarily the Brazilian legal context. In this way, we try to explain how the civil law and its legislation is used in a Brazilian court, in judgements about moral damage, especially in deliberations with the three or more judges. Finally, in the third part (about a controversial media case) we present a case study to show how the agreement that we called the "dissonant agreement" takes place in a real situation of interaction between magistrates in a Brazilian court in a judgment of moral damage ("the insulting title's case"). We have analyzed three transcribed excerpts of the judgment to demonstrate how the management of the disagreement takes place all long that deliberation. The conclusion of the work shows possibilities to be "potentially persuasive" (Aristotle) in a legal deliberation, and the way magistrates address to their interlocutors in a judgement among other legal experts. The careful analysis of the interaction among judges during the deliberations recorded is useful to demonstrate the rhetorical dimension of a deliberation and the kind of negotiation that may be made in these situations, where pain and suffering of others are being judged in moral damage cases. Even if the situations analysed do not have an argumentative purpose declared, it might be observed that the speakers tried to adopt strategies to make prevail their conclusions, that is, the amount to be paid, in opposition of those of their colleagues in session, like we tried to show in the analysis. Thus, we believe that the excerpts provide interesting ways to examine the importance of analysing argumentation in context of interaction (Plantin, 2015). In this way, we have looked more closely the process of mitigating the disagreement expressed by magistrates in a polemic legal deliberation. To conclude, we tried, in this analysis, to highlight the strategic dimension of the rhetorical disagreement among magistrates during a polemical trial in a media case, on a legal court in Brazil. As we try to show, the called "dissonant agreement" - or the subtle concessive movement - is an interesting face of the argumentative and rhetorical movement in a legal court in Brazil.

INDEX

Keywords : disagreement, argumentation, strategy, moral damage, media

Mots-clés : désaccord, argumentation, stratégie, dégâts moraux, médias

\section{AUTEUR}

\section{RUBENS DAMASCENO-MORAIS}

Université de Goiás - UFG / Brésil

r.damasceno.morais@uol.com.br 